\title{
SOLUCIÓN DE CONTROVERSIAS INVERSOR-ESTADO EN LOS NUEVOS TRATADOS DE INTEGRACIÓN REGIONAL: EL CASO DEL ACUERDO DE ASOCIACIÓN TRANSPACÍFICO
}

\section{SOLUCC̃O DE DISPUTAS INVESTIDOR-ESTADO NOS NOVOS ACORDOS DE INTEGRACCÃO REGIONAL: O CASO DO ACORDO DE ASSOCIAÇÃO TRANSPACÍFICO}

Magdalena Bas Vilizzio*

Resumen: En la actualidad el Derecho de la integración comprende la regulación de áreas que dos décadas atrás conformaban sistemas regulados en paralelo, en particular el comercio intrazona y la protección $y$ promoción de inversiones, entre otros temas. Asimismo, el concepto de región se ha flexibilizado, englobando situaciones diversas como las de región ampliada o tratados mega regionales.

En este marco, el presente artículo tiene como objetivo el análisis del Acuerdo de Asociación Transpacífico, en vistas que tres Estados Latinoamericanos son partes signatarias: Chile, México y Perú. Se opta por el estudio particular de la Sección B del Capítulo 9 relativo a la solución de controversias inversor-Estado, como tema típico de los tratados de integración de última generación. Se parte de un análisis crítico de los principales aspectos procesales del arbitraje ad hoc en los foros externos a los cuales remite, así como las excepciones más importantes, por ejemplo las medidas de control del tabaco, una innovación en los acuerdos económicos.

El trabajo cierra con las reflexiones finales, entre las cuales se destaca que si bien el Acuerdo TPP presenta innovaciones que implican avances en la reforma del sistema, en gran medida replica disposiciones de otros tratados comerciales $y / o$ de inversiones dejando sin resolver gran parte de

\footnotetext{
* Profesora Adscripta de Derecho Internacional Público de la Universidad de la República y Profesora Titular de Derecho del Comercio Internacional (Maestría en Comercio Internacional e Integración) de la Universidad de Montevideo. Doctoranda en Relaciones Internacionales de la Universidad Nacional de la Plata. Miembro de la Red de Juristas para la Integración Latinoamericana y la Red Latinoamericana de Derecho Económico Internacional. E-mail: magdalenabas@gmail.com
} 
los problemas existentes.

Resumo: Na atualidade, o Direito da integração abrange o regulamento de áreas que duas décadas atrás configuravam diferentes sistemas regulados de maneira paralela, especialmente o comércio intrazona e a proteção e promoção de investimentos, dentre outros temas. Ainda, o conceito de região se tem flexibilizado abarcando situações diversas, tais como a de região ampliada ou tratado mega regional.

Neste contexto, o presente artigo tem como objetivo a análise do Acordo de Associação Transpacífico, devido a que três Estados latino-americanos são partes signatárias do Acordo: Chile, México e Peru. Opta-se por estudar, especificamente, a Seção $B$ do Capítulo 9 relativo à solução de controvérsias investidor-Estado, por ser esta uma questão característica dos tratados de integração da última geração. Em primeiro lugar, realiza-se uma análise crítica dos principais aspectos processuais da arbitragem ad hoc nos foros externos aos quais se remete, bem como das exceções mais importantes, tais como as medidas de controle do tabagismo, que representam uma inovação nos acordos econômicos.

$O$ trabalho se encerra com reflexões finais, entre as quais se destaca que apesar de apresentar inovações que acarretam avanços na reforma do sistema, o Acordo, em grande medida, só replica disposições de outros tratados comerciais e/ou de investimentos, não resolvendo, desta maneira, a maioria dos problemas existentes.

Palabras clave: Derecho de la integración, Inversiones, Solución de controversias, Acuerdo de Asociación Transpacífico, TPP

Palavras-chave: Direito da integração, Investimentos, Solução de disputas, Acordo de Associação Transpacífico, TPP

\section{INTRODUCCIÓN}

La integración es un proceso por el cual dos o más Estados, como entidades soberanas, toman la decisión soberana de agruparse con el fin de formar nuevas instituciones supranacionales o coordinar sus políticas en el marco de instituciones intergubernamentales. Dicho fenómenos tiene como piedra fundamental uno o más acuerdos internacionales donde, en general, se establecen los objetivos del sistema, sus principios fundamentales, su estructura orgánica provisoria o definitiva, disposiciones relativas al sistema normativo derivado de sus órganos, y un sistema de solución de diferencias con órganos ad hoc o permanentes.

Hasta hace dos décadas aproximadamente, los tratados de integración se celebran entre Estados con proximidad geográfica y procuran alcanzar un objetivo pura o mayoritariamente comercial, enmarcado en el sistema multilateral de comercio del Acuerdo General de 
Aranceles y Comercio (GATT) y la Organización Mundial del Comercio (OMC). Por tanto, suelen ser denominados por la doctrina como acuerdos comerciales regionales o acuerdos preferenciales regionales. En el mismo período, el sistema de tratados de integración convive con un sistema de acuerdos internacionales cuyo objeto es la promoción y protección de inversiones, conformado por los tratados bilaterales de inversión (TBI) y el Centro Internacional de Arreglo de Diferencias relativas a Inversiones (CIADI) como principal foro al que remiten los TBI en caso de disputa entre inversores y Estados (sistema TBI-CIADI) ${ }^{1}$.

En suma, hasta mediados de los noventa, los Estados se encontraban obligados internacionalmente por un entramado de acuerdos económicos compuesto por dos sistemas paralelos, figura que Álvarez denomina "sopa de espaguetis", una versión más compleja de la idea de Bhagwati del "tazón de espaguetis" de los tratados de libre comercio (TLC), una de las modalidades de los acuerdos comerciales regionales.

Dos nuevas características irrumpen en los acuerdos de integración entrada la década del noventa. En primer lugar el ámbito temático a ser regulado es extendido, excediendo el ámbito del sistema GATT-OMC, extendiéndose a nuevas áreas que incluyen la promoción y protección de inversiones así como los asuntos laborales, las medidas anticorrupción, el medio ambiente, derechos humanos, entre otros.

En segundo lugar, se flexibiliza o desaparece el concepto de región como marco geográfico en el que se desarrollan los procesos de integración. De esta manera se da paso a tratados entre Estados sin o con escasa proximidad geográfica como el Acuerdo Transpacífico de Asociación Económica Estratégica o P4 entre Chile, Nueva Zelanda, Singapur y Brunei celebrado en 2005; tratados interregionales o entre Estados y regiones, como el actualmente en negociación Acuerdo Económico y Comercial Global entre Canadá y la Unión Europea (UE); y tratados en el marco de regiones ampliadas o mega regionalismo, como el Acuerdo de Asociación Transpacífico (Acuerdo del TPP por su sigla en inglés) firmado en 2016.

En vistas de lo anterior, y teniendo en mente que tres Estados Latinoamericanos son parte del Acuerdo del TPP, el presente trabajo tiene como objetivo aportar al Derecho de la integración latinoamericana mediante el análisis del mencionado acuerdo, en particular las disposiciones relativas al sistema de solución de controversias inversor-

1 Para un análisis específico de este punto en relación a los Estados Sudamericanos ver: BAS VILIZZIO, Magdalena. "Solución de controversias en los tratados bilaterales de inversión: mapa de situación en América del Sur" [consultado 01.07.16]. Revista de la Secretaría del Tribunal Permanente de Revisión. 2015, año 3, no 5, Marzo 2015, 233 - 253. Disponible en: <http://www. revistastpr.com/index.php/rstpr/article/view/120/pdf>

2 ALVAREZ, José A. "Is the Trans-Pacific Partnership's investment chapter the new "gold standard"?" [en línea]. Working Paper IILJ 2016/3 MegaRed Series. Nueva York: New York University, School of Law, 2016, p. 14 [consultado 01.07.16]. Disponible en: <https://wp.nyu. edu/megareg/wp-content/uploads/sites/3134/2016/03/Alvarez_IILJ-MegaReg_2016-3.pdf> 
Estado puesto que presenta importantes excepciones para los Estados Latinoamericanos (Sección B del Capítulo 9).

\section{EL TPP EN EL SISTEMA INTERNACIONAL: ASPECTOS GENERALES}

Tras casi seis años de negociaciones, el 4 de febrero de 2016, doce Estados con costas al Océano Pacífico, a saber: Australia, Brunei, Canadá, Chile, Estados Unidos, Japón, Malasia, México, Nueva Zelanda, Perú, Singapur y Vietnam, firman el tratado constitutivo del TPP en Auckland, Nueva Zelanda. Su embrión es el Acuerdo Transpacífico de Asociación Económica Estratégica, también conocido como P4, entre Brunei, Chile, Nueva Zelanda y Singapur del 18 de julio de $2005^{3}$, en cuyo artículo 20.6 establece que "estará abierto a adhesión para cualquier Economía de APEC u otro Estado, en los términos que acuerden las Partes"' . De esta manera, en el año 2008 Estados Unidos se convierte en el primer Estado en participar en las negociaciones del P4, inicialmente en materia de inversión y servicios y posteriormente en anunciar su intención de participar del TPP.

Las más de seiscientas páginas del acuerdo se estructuran en un preámbulo y treinta capítulos que regulan: 1 . Disposiciones iniciales; 2 . Comercio de bienes (acceso a mercado y agricultura); 3 . Reglas de origen y procedimientos relativos al origen; 4. Textiles; 5. Administración Aduanera y Facilitación de Comercio; 6. Defensa comercial; 7. Medidas Sanitarias y Fitosanitarias (MSF); 8. Obstáculos Técnicos al Comercio (OTC); 9. Inversiones; 10. Comercio Transfronterizo de Servicios; 11. Servicios Financieros; 12. Entrada Temporal de Personas de Negocios; 13. Telecomunicaciones; 14. Comercio Electrónico; 15. Contratación Pública; 16. Competencia; 17. Empresas del Estado y monopolios designados; 18. Propiedad Intelectual; 19. Asuntos Laborales; 20. Medio Ambiente; 21. Cooperación; 22. Competitividad; 23. Desarrollo; 24. Pequeñas y Medianas Empresas; 25. Coherencia regulatoria; 26. Transparencia y Anticorrupción; 27. Administración y Disposiciones Institucionales; 28. Solución de Diferencias; 29. Excepciones; 30. Disposiciones Finales. Asimismo deben sumarse aproximadamente dos mil páginas de anexos 5 .

3 Dado que los signatarios depositaron los instrumentos de ratificación con posterioridad a 1 de enero de 2006, el P4 entró en vigor para cada uno de ellos 30 días después de la fecha de depósito de los mencionados instrumentos (artículo 20.4 numeral 3). Esto es, para Nueva Zelanda y Singapur el acuerdo entra en vigor el 28 de mayo de 2006, para Brunei el 12 de julio de 2006, y finalmente para Chile el 8 de noviembre de 2006.

4 APEC es el Foro de Cooperación Económica de Asia Pacífico. Sus miembros son: Australia, Brunei, Canadá, China, Estados Unidos, Filipinas, Hong Kong, Indonesia, Malasia, México, Nueva Zelanda, Papúa Nueva Guinea, Perú, República de Corea, Rusia, Singapur, Taipéi Chino, Tailandia, Vietnam.

5 Conforme al artículo 30.5.1 el tratado entrará en vigor sesenta días después de la fecha en 
Como se indicara anteriormente, el acuerdo del TPP cumple con las dos características de los nuevos tratados de integración, esto es, vinculan a Estados de una región ampliada y extienden su ámbito de regulación a una multiplicidad de temáticas, muchas de ellas fuera de la órbita del sistema multilateral de comercio. De esta forma, temas habitualmente regulados por fuera del ámbito del Derecho de la integración, como ser la promoción y protección de inversiones, pasan a ser parte integrante del tratado fundamental del bloque.

En los siguientes puntos, este trabajo se encargará del análisis particular del sistema de solución de controversias inversor-Estado previsto en la Sección B del Capítulo 9 (Inversiones), uno de los capítulos más controvertidos del tratado bajo estudio.

\section{SOLUCIÓN DE CONTROVERSIAS INVERSOR-ESTADO EN EL TPP: ¿INNOVACIÓN O REPETICIÓN?}

\subsection{El Capítulo de Inversión del TPP en la "sopa de espagueti"}

El capítulo 9 del acuerdo del TPP, dedicado a la regulación de la promoción y protección de inversiones recíprocas, se suma a la red de acuerdos entre los Estados parte, ya sean TBI o TLC que cuentan con capítulos de inversiones. Específicamente la Sección B, referente al sistema de solución de controversias entre inversores de un Estado parte y Estados parte receptores de la inversión, se solapa con el entramado de relaciones que muestran las tablas 1 y 2 . Se excluyen de la sistematización realizada los tratados cuya vigencia se encuentra pendiente y los terminados, así como aquellos que, a pesar de contar con disposiciones que regulan la inversión extranjera, no regulan la solución de controversias inversor-Estado.

\footnotetext{
la que todos los signatarios originales hayan depositado el instrumento de ratificación ante Nueva Zelanda. Si en un plazo de dos años, los doce Estados signatarios originales no hubieran realizado el depósito, el tratado entrará en vigor sesenta días después de dicho período si al menos seis Estados depositaron sus instrumentos de ratificación y en conjunto alcanzan $s$ suman al menos el 85 por ciento del producto interno bruto a 2013 (artículo 30.5.2). El acuerdo prevé una tercera posibilidad que es la entrada en vigor a los sesenta días del sexto depósito de un instrumento de ratificación de los signatarios originales, siempre que alcancen el 85 por ciento del producto interno bruto a 2013 (artículo 30.5.3).
} 
Tabla 1: Entramado de relaciones derivadas de los TBI en vigor entre los miembros del TPP, que regulan la solución de controversias inversor-Estado.

\begin{tabular}{|c|c|c|c|c|c|c|c|c|c|c|c|c|c|}
\hline & Australia & Brunei & Canadá & Chile & $\begin{array}{l}\text { Estados } \\
\text { Unidos } \\
\end{array}$ & Japón & Malasia & México & $\begin{array}{l}\text { Nueva } \\
\text { Zelanda }\end{array}$ & Perú & Singapur & Vietnam & TOTAL \\
\hline Australia & - & & & & & & & & & & & & 2 \\
\hline Brunei & & - & & & & & & & & & & & 0 \\
\hline Canadá & & & - & & & & & & & & & & 1 \\
\hline Chile & & & & - & & & & & & & & & 2 \\
\hline $\begin{array}{l}\text { Estados } \\
\text { Unidos }\end{array}$ & & & & & - & & & & & & & & 0 \\
\hline Japón & & & & & & - & & & & & & & 1 \\
\hline Malasia & & & & & & & - & & & & & & 3 \\
\hline México & & & & & & & & - & & & & & 2 \\
\hline $\begin{array}{l}\text { Nueva } \\
\text { Zelanda }\end{array}$ & & & & & & & & & - & & & & 0 \\
\hline Perú & & & & & & & & & & - & & & 4 \\
\hline Singapur & & & & & & & & & & & - & & 2 \\
\hline Vietnam & & & & & & & & & & & & - & 4 \\
\hline TOTAL & 3 & 0 & 1 & 1 & 0 & 2 & 3 & 1 & 0 & 4 & 2 & 4 & 21 \\
\hline
\end{tabular}

Elaboración propia en base a datos del Investment Hub de la UNCTAD, actualizados al 9 julio de 2016. 
Tabla 2: Entramado de relaciones derivadas de TLC en vigor entre los miembros del TPP, que regulan la solución de controversias inversor - Estado.

\begin{tabular}{|c|c|c|c|c|c|c|c|c|c|c|c|c|c|}
\hline & Australia & Brunei & Canadá & Chile & $\begin{array}{l}\text { Estados } \\
\text { Unidos }\end{array}$ & Japón & Malasia & México & $\begin{array}{c}\text { Nueva } \\
\text { Zelanda }\end{array}$ & Perú & Singapur & Vietnam & TOTAL \\
\hline Australia & - & & & & & & & & & & & & 7 \\
\hline Brunei & & - & & & & & & & & & & & 6 \\
\hline Canadá & & & - & & & & & & & & & & 4 \\
\hline Chile & & & & - & & & & & & & & & 6 \\
\hline $\begin{array}{l}\text { Estados } \\
\text { Unidos }\end{array}$ & & & & & - & & & & & & & & 6 \\
\hline Japón & & & & & & - & & & & & & & 2 \\
\hline Malasia & & & & & & & - & & & & & & 6 \\
\hline México & & & & & & & & - & & & & & 4 \\
\hline $\begin{array}{l}\text { Nueva } \\
\text { Zelanda }\end{array}$ & & & & & & & & & - & & & & 5 \\
\hline Perú & & & & & & & & & & - & & & 6 \\
\hline Singapur & & & & & & & & & & & - & & 7 \\
\hline Vietnam & & & & & & & & & & & & - & 5 \\
\hline TOTAL & 7 & 6 & 4 & 6 & 6 & 2 & 6 & 4 & 5 & 6 & 7 & 5 & 64 \\
\hline
\end{tabular}

Elaboración propia en base a datos del Investment Hub de la UNCTAD, actualizados al 9 julio de 2016. 


\subsection{Solución de controversias inversor-Estado: aspectos procesales}

La Sección B del Capítulo de Inversión se ocupa de las controversias surgidas por reclamos de inversores extranjeros de nacionalidad de alguno de los miembros del TPP frente a otros miembros que sean receptores de la inversión. Si bien se trata de una sección extensa, compleja y rodeada de una serie de excepciones, en este apartado se intenterá realizar un estudio de sus principales artículos, en particular aquellos que han generado mayor discusión doctrinaria, y en el siguiente apartado se realizará un análisis crítico de las principales excepciones al sistema.

En primer lugar, el proceso de resolución de disputas comienza con un período de enfriamiento (cooling-off period) por un plazo de seis meses. Durante este, las partes deben intentar resolver la controversia mediante consultas y negociación, con o sin la participación no vinculante de buenos oficiantes o mediadores (artículo 9.18). La solución amigable suele ser habitual en los TBI y/o capítulos de inversiones previstos en los TLC, como etapa previa obligatoria al sometimiento de la controversia al arbitraje internacional.

En este sentido, una vez cumplido el período de enfriamiento y de no haberse arribado a una solución mutuamente convenida, queda abierta la vía arbitral. El inicio de la vía arbitral debe cumplir con dos requisitos: 1) requisito ratione personae: el inversor debe tener nacionalidad de uno de los Estados parte del Acuerdo TPP, y debe actuar en nombre propio o por cuenta de una empresa en el país del demandado bajo su control directo o indirecto; 2) requisito ratione materiae: el reclamo debe basarse en una materia de naturaleza jurídica, esto es, un caso de incumplimiento, por parte del Estado receptor de la inversión, de compromisos explicitados en la Sección A del capítulo, una autorización de inversión o un contrato de inversión, que le generó daño económico al demandante (artículo 9.19.1).

Es el demandante quien tiene la opción de foro (forum shopping) entre el arbitraje ad hoc en el CIADI si los Estados son parte del Convenio de Washington de 1965 (ver tabla 3); el mecanismo complementario del CIADI para los Estados que no sean parte del mencionado convenio; las Reglas de arbitraje de la Comisión de las Naciones Unidas para el Derecho Mercantil Internacional (CNUDMI); cualquier otra institución arbitral u otras reglas de arbitraje a su elección (artículo 9.19.4). Sea cual sea el foro elegido, el solo sometimiento del reclamo a la vía arbitral implica que los Estados signatarios han otorgado su consentimiento de prorrogar la jurisdicción a favor del foro en cuestión, conforme a las normas aplicables a cada uno de los casos (artículo 9.20).

Es importante tener en cuenta que se trata de instituciones externas 
al TPP y que no necesariamente responden a las lógicas jurídicas del conjunto de naciones de una vasta región como la transpacífica. En otras instancias en América del Sur se ha intentado la regulación de un sistema de solución de controversias inversor-Estado propio de la región, tal es el caso del Centro de Solución de Controversias en Materia de Inversiones de la Unasur, cuyo protocolo constitutivo se encuentra actualmente en negociación ${ }^{6}$.

Tabla 3: Miembros del TPP que son parte del Convenio de Washington de 1965

\begin{tabular}{|l|l|l|l|}
\hline \multicolumn{1}{|c|}{ Estado } & \multicolumn{1}{|c|}{ Firma o adhesión } & \multicolumn{1}{c|}{$\begin{array}{c}\text { Depósito de ratifi- } \\
\text { cacion }\end{array}$} & \multicolumn{1}{c|}{ Entrada en vigor } \\
\hline Australia & 24 marzo 1975 & 2 mayo 1991 & 1 junio 1991 \\
\hline Brunei & 16 setiembre 2002 & 16 setiembre 2002 & 16 octubre 2002 \\
\hline Canadá & 15 diciembre 2006 & 1 noviembre 2013 & 1 diciembre 2013 \\
\hline Chile & 25 enero 1991 & 24 setiembre 1991 & 24 octubre 1991 \\
\hline Estados Unidos & 27 agosto 1965 & 10 junio 1966 & 14 octubre 1966 \\
\hline Japón & 23 setiembre 1965 & 17 agosto 1967 & 16 setiembre 1967 \\
\hline Malasia & 22 octubre 1965 & 8 agosto 1966 & 14 octubre 1966 \\
\hline México & - & - & - \\
\hline Nueva Zelanda & 2 setiembre 1970 & 2 abril 1980 & 2 mayo 1980 \\
\hline Perú & 4 setiembre 1991 & 9 agosto 1993 & 8 setiembre 1993 \\
\hline Singapur & 3 febrero 1968 & 14 octubre 1968 & 13 noviembre 1968 \\
\hline Vietnam & - & - & - \\
\hline
\end{tabular}

Elaboración propia en base a datos del CIADI, actualizados al 9 julio de 2016

Por otra parte, el texto no prevé el agotamiento previo de la jurisdicción doméstica como requisito para el inicio de la vía arbitral internacional, en contra de las ideas de la doctrina Calvo (1868) respecto a la obligación de los extranjeros de agotar los recursos internos previo a la protección o intervención diplomática del Estado de su nacionalidad ${ }^{7}$. Asimismo, debe destacarse que la entrada en vigor del acuerdo TPP entrañaría la existencia de un nuevo instrumento jurídico en el entramado internacional que con cuente con tal requisito. Por tanto, las mayores ventajas podrían ser invocadas por los inversores de terceros

6 Información disponible en: www.nodal.am/2016/01/expertos-de-unasur-ultiman-detallespara-creacion-de-tribunal-o-comision-parasolucion-de-controversias-sobre-inversiones/ (Consultado: 05.07.16)

7 TAMBURINI, Francesco. "Historia y Destino de la Doctrina Calvo: ¿Actualidad u obsolescencia del pensamiento de Carlos Calvo?". Revista de estudios histórico-jurídicos. 2002, n XXIV, p. 82 [consultado 23.01.15]. Disponible en: <http://www.rehj.cl/index.php/rehj/article/ view/363/343> 
Estados en función de una cláusula de la nación más favorecida prevista otro tratado que vincule a un tercer Estado con un Estado parte del TPP, aun en los casos que el requisito sea obligatorio ${ }^{8}$.

Tampoco se prevé la jurisdicción doméstica como opción junto al arbitraje internacional a la hora de resolver las disputas entre inversores y Estados. No obstante, existe una excepción respecto a los tres Estados parte Latinoamericanos, Chile, México y Perú, junto a Vietnam, en el sentido que si un inversor extranjero decide presentar un reclamo ante sus tribunales administrativos o judiciales internos, tal elección es definitiva y exclusiva. Por tanto, el inversor no tiene la posibilidad de someter el caso ante tribunales arbitrales internacionales, teniendo por ende una segunda oportunidad de resolución (Anexo 9-J).

Otro elemento a destacar es el principio de transparencia y publicidad de los diferentes documentos, actuaciones y audiencias a lo largo del proceso arbitral, salvo que se trata de información confidencial cuya divulgación deberá ser protegida (artículo 9.24). Esta disposición demuestra la evolución positiva que el sistema ha tenido a lo largo del tiempo, especialmente en relación al reconocimiento a las normas internas que consagran al derecho de acceso a la información pública y que, de acuerdo a Tung, podrá generar mayor conocimiento social de casos controversiales ${ }^{9}$. En la vereda opuesta, Johnson y Sachs afirman que se trata de un paso atrás en relación a los principios de publicidad presentes en los modelos de acuerdos comerciales de Estados Unidos. Asimismo, entienden que aun deja afuera a los diferentes interesados en el proceso, cuya única vía de acceso continúa siendo la presentación de un escrito como amicus curiae, cuya aceptación es potestad del tribunal arbitral que entienda en el caso concreto ${ }^{10}$.

Finalmente es innegable el avance en relación al Código de Conducta al que estarán sujetos los árbitros (artículo 9.22.6), siendo de aplicación las disposiciones generales sobre solución de controversias del Acuerdo TPP (capítulo 28). De acuerdo a Johnson y Sachs, los árbitros internacionales no suelen tener los mismos requisitos de imparcialidad e independencia que los jueces en los sistemas judiciales domésticos, y de hecho, gran parte de las críticas al sistema de solución

8 Para un análisis más profundo de la cláusula de la nación más favorecida en la solución de controversias inversor-Estado ver: BANIFATEMI, Yas . "The emerging jurisprudence on the Most-Favored-Nation Treatment in investment arbitration". En: BJORKLUND, Andrea (ed.). Investment Treaty Law. Current Issues III - Remedies in International Investment Law: Emerging Jurisprudence of International Investment Law. Londres: BIICL, 2009, p. 241-273.

9 TUNG, Ko-Yunng. "Investor-State dispute settlement under the Trans-Pacific Partnership". The California International Law Journal. 205, vol 23, no 1, p. 22 [consultado 01.07.16]. Disponible en: <http://www.mofo.com/ /media/Files/Articles/2015/08/150800InvestorStateD isputeSettlement.pdf $>$

10 JOHNSON, Lise y SACHS, Lisa. “The TPP’s investment chapter: entrenching, rather and reforming, a flawed system” [en línea]. CCSI Policy Paper. Columbia Center on Sustainable Investment [consultado 01.07.16]. Disponible en: <http://ccsi.columbia.edu/files/2015/11/TPPentrenching-flaws-21-Nov-FINAL.pdf> 
de controversias inversor-Estado radican en este punto. Por tanto la iniciativa es un punto a destacar, pero debe tomarse con prudencia a la espera de su efectiva elaboración por los signatarios del acuerdo ${ }^{11}$.

\subsection{Las excepciones a la solución de controversias inversor- Estado}

\subsubsection{Las excepciones son la regla}

La sabiduría popular indica que "la excepción hace la regla". No obstante, las excepciones en el Acuerdo del TPP son precisamente la regla, no la excepción. Como explica Bollyky las excepciones están presentes en todos los capítulos incluso en el preámbulo, bajo diferentes formas, como ser excepciones a principios generales, excepciones a excepciones, exclusiones explícitas o implícitas, aclaraciones, limitación de la normativa aplicable, etc. ${ }^{12}$.

En materia de solución de controversias inversor-Estado además de la excepción anteriormente analizada relativa a la jurisdicción doméstica como opción para la resolución de disputas en el caso de Chile, México, Perú y Vietnam (Anexo 9-J), las excepciones más destacables son las medidas de control del tabaco (artículo 29.5) y la deuda soberana (Anexo 9-G).

\subsubsection{Medidas de control del tabaco}

El artículo, 29.5 en el Capítulo Excepciones y Disposiciones Generales, prevé la que tal vez sea la disposición más innovadora de todo el Acuerdo TPP, y sin duda la excepción más importante del sistema de solución de controversias inversor-Estado en el acuerdo. La norma indica que un Estado parte puede optar por que las medidas de control de tabaco que decida tomar no sean susceptibles de reclamaciones en el marco de la Sección B del Capítulo 9, y por tanto un inversor nacional de otro Estado parte no pueda iniciar un arbitraje por tales motivos. La opción puede ser ejercida en cualquier momento, incluso al inicio o durante el proceso arbitral.

La inclusión de esta disposición responde a las características especiales del tabaco, como único bien de consumo que cuenta con un tratado internacional que controla y previene su consumo ${ }^{13}$, el Convenio Marco de la Organización Mundial de la Salud para el Control

11 Ibíd., p. 8.

12 BOLLYKY, THOMAS J. "TPP tobacco exception proves the new rule in trade. Council for Roreign Relations" [en línea]. Expert Brief, 4 de febrero de 2016. [consultado 09.07.16]. Disponible en: <http://www.cfr.org/trade/tpp-tobacco-exception-proves-new-rule-trade/ p37509>

13 Ibíd. 
del Tabaco, celebrado el 21 de mayo de 2003 y en vigor desde el 27 de febrero de 2005, que cuenta con 180 Estados parte ${ }^{14}$.

Asimismo, Australia y Nueva Zelanda fueron dos de los Estados parte del Acuerdo TPP que más presionaron para la inclusión de la excepción del control del tabaco ${ }^{15}$. El primer Estado tiene la experiencia de la demanda de la tabacalera Philip Morris en la Corte Permanente de Arbitraje (caso 2012-2), en base a la Ley de empaquetado genérico de los cigarrillos (2011) y sus normas modificatorias y complementarias.. El argumento del inversor se basaba en la supuesta violación de las disposiciones del TBI Hong Kong - Australia relativas al trato justo y equitativo y expropiación de la propiedad intelectual. El 17 de diciembre de 2015, el tribunal aceptó la objeción de Australia en relación a que constituye abuso de derecho que el demandante realizara una restructuración en Hong Kong, poco antes del inicio del arbitraje ${ }^{16}$.

Un arbitraje similar es el iniciado por Philip Morris Brand Sàrl, Philip Morris Products S.A. y Abal Hermanos S.A. contra Uruguay (CIADI caso $\mathrm{ARB} / 10 / 7$ ) por entender que las medidas relativas a la presentación única por marca comercial y el uso de pictogramas con advertencias sanitarias del $80 \%$ del frente y el reverso de las cajillas, representaban un incumplimiento del TBI Uruguay-Suiza ${ }^{17}$. El 8 de julio de 2016, el tribunal arbitral ad hoc emite un laudo que no hace lugar a las pretensiones de la tabacalera, reconociendo el poder soberano de policía del Estado para regular la salud pública, y condenando a la empresa al pago de los gastos del tribunal arbitral y administrativos del CIADI, así como un reintegro de parte de los costos pagados por Uruguay ${ }^{18}$.

Adicionalmente, la situación de Nueva Zelanda es prueba fehaciente que, como explica de Zayas ${ }^{19}$, las demandas o amenazas de demandas de manos de inversores extranjeros pueden derivar en una

\footnotetext{
14 Información disponible en el sitio web de la Organización Mundial de la Salud: <http:// www.who.int/fctc/signatories_parties/es/> [consultado 10.07.16].

15 Independientemente del impulso de Australia y Nueva Zelanda en este punto, son interesantes los cambios que este tratado implica a la posición de ambos frente a la solución de controversias inversor- Estado. Según Álvarez, ambos países optaron por excluirla de los TBI celebrados desde el año 2011 (año en que se oficializa la demanda de Philip Morris Asia. ALVAREZ. Op. cit., p. 31.

16 Laudo de jurisdicción y admisibilidad del 17 de diciembre de 2015 disponible en: <https:// pcacases.com/web/sendAttach/1711> [consultado 16.05.16].

17 Un análisis del caso previo a la emisión del laudo puede verse en: BAS VILIZZIO, Magdalena. "Arbitraje en el CIADI: algunas reflexiones en torno al caso Philip Morris contra Uruguay". En: TRAVIESO, Juan Antonio y CAMPI, Germán (ed.). Derecho Internacional Público. Su ingeniería y arquitectura en el siglo XXI. Buenos Aires: Editorial Albremática - elDial.com, 2016, p. 551-562; y MICHELINI, Felipe. "The request for arbitration submitted by Philips Morris International vs Uruguay: a human rights perspective”. Simposio Common Grounds New Zealand - América Latina - A legal Perspective (22 de octubre de 2015). Wellington: The Law School Victoria University, 2015.

18 Laudo del 8 de julio de 2016 disponible en: <https://medios.presidencia.gub.uy/tav portal/2016/noticias/NO_U130/laudo_spal.pdf > [consultado 08.07.16].

19 Experto Independiente sobre la promoción de un orden internacional democrático y equitativo nombrado por el Consejo de Derechos Humanos de la Organización de Naciones Unidas.
} 
"parálisis normativa" o incluso una "congelación", impidiendo a los Estados adoptar reglamentos en áreas tales como la salud pública ${ }^{20}$. En este caso concreto, Nueva Zelanda paraliza la sanción de una norma de empaque genérico de cigarrillos hasta tanto no fuera emitido el laudo del arbitraje contra Australia. Un mes después de concluido el caso, el Primer Ministro neozelandés anuncia que prevé que la ley de empaque genérico sea sancionada a fines de $2016^{21}$.

Johnson y Sachs señalan que la excepción de las medidas de control del tabaco es necesaria para proteger a los Estados de reclamos como los anteriormente indicados, pero que el acuerdo no se pronuncia respecto a otras áreas objeto de políticas públicas soberanas, como ser el medio ambiente, la salud o la seguridad pública ${ }^{22}$. De esta manera la soberanía estatal puede verse limitada y, en palabras de Arato, posicionar a las empresas multinacionales como creadoras de normas jurídicas ${ }^{23}$.

\subsubsection{Deuda pública}

El Anexo 9 - G establece que dado que la compra de deuda soberana es una empresa de riesgo, no podrán emitirse laudos arbitrales a favor del demandante en caos de reclamos por incumplimiento o falta de pago de títulos de deuda soberana emitida por una de las Partes del acuerdo. La excepción a la excepción la constituyen las violaciones a los compromisos emanados de la Parte A del Capítulo de Inversiones, estando la carga de la prueba sobre los hombros del demandante.

Diferente es el caso de la reestructuración de deuda, dado que no pueden ser sometidos a un arbitraje internacional, o continuar en uno si ya fueron sometidos, siempre se trate de una reestructuración negociada al momento o después del sometimiento. La norma no explicita si la disposición incluye aquellos casos en que los títulos de deuda no establezca cláusulas de acción colectiva ${ }^{24}$, y una parte de los

20 ONU. Cuarto reporte del Experto Independiente sobre la promoción de un orden internacional democrático y equitativo. Documento A/70/285 [en línea]. Nueva York: ONU, 2015, p. 5 [consultado 02.02.16]. Disponible en: <http://www.un.org/en/ga/search/ view_doc.asp?symbol=A/70/285\&referer=http://www.ohchr.org/EN/Issues/IntOrder/Pages/ IEInternationalorderIndex.aspx\&Lang=S $>$

21 MCHAFFIE, Kate. "New Zealand to implement plain packaging for tobacco"[en línea]. Lexology, 18 de febrero de 2016. [consultado 09.05.2016]. Disponible en: <http://www.lexology. com/library/detail.aspx?g=2f267230-efb7-4348-bb47-ee53c89bd601>

22 JOHNSON, Lise y SACHS, Lisa. Op. cit., p. 3.

23 ARATO, Julián. “Corporations as Lawmakers”. Harvard International Law Journal. 2015, vol $56, n^{\circ} 2$, p. 283.

24 Las cláusulas de acción colectiva establecen que una mayoría calificada determinada de acreedores tiene la potestad de modificar los términos más importantes previstos en los títulos de deuda soberana, obligando a la totalidad de acreedores, de forma de impedir las acciones individuales de acreedores disidentes. RANIERI, Agustina. "Deuda soberana: problemas y soluciones en la encrucijada” [en línea]. Revista Pensar en Derecho. 2015, no 6, p. 245-304 [consultado 05.06.16]. Disponible en: <http://www.derecho.uba.ar/publicaciones/pensar-enderecho/revistas/6/deuda-soberana-problemas-y-soluciones-en-la-encrucijada.pdf > 
acreedores estén habilitados para decidir no ingresar en el proceso de reestructuración (acreedores disidentes o holdouts). Por tanto cabe preguntarse si la norma abarca o no a los acreedores disidentes.

\section{REFLEXIONES FINALES}

A lo largo de este trabajo se analizaron las disposiciones sobre solución de controversias inversor-Estado incluidas en el Acuerdo TPP, acuerdo de integración que en sus cerca de 2700 páginas además de regular aspectos relativos al comercio de bienes y servicios alcanza otras áreas como la promoción y protección de inversiones. En consecuencia se superpone con un entramado de acuerdos comerciales regionales y TBI que regulan las relaciones entre los Estados parte.

El sistema de solución de controversias inversor-Estado previsto replica en gran medida las disposiciones de los TBI o los capítulos contenidos en los TLC celebrados por Estados Unidos en los últimos años, a excepción de algunas innovaciones. En particular es de destacar que no se opta por un mecanismo propio del proceso de integración, como en el caso de la Unasur o más recientemente de los Tribunales de inversiones de primera instancia y de apelación en el marco del Acuerdo de la Asociación Transatlántica de Comercio e Inversión entre Estados Unidos y la $\mathrm{UE}^{25}$ o el Acuerdo Económico y Comercial Global entre la UE y Canadá ${ }^{26}$.

El capítulo 9 del Acuerdo TPP se limita a indicar un elenco de foros externos ante los cuales el demandante puede efectivizar su reclamo. No se presentan cambios sustantivos, ni se resuelven los cuestionamientos respecto a la solución de disputas por parte de tribunales ad hoc y por ende sin permanencia, que no han generado una jurisprudencia uniforme, ni cuentan con la posibilidad de apelación por un órgano superior. Por tanto, las diferentes propuesta de reforma al sistema ${ }^{27}$ no han sido tenidas en cuenta por los autores del tratado,

Si bien la excepción de las medidas de control del tabaco es una innovación que sería deseable que sea replicada en futuros acuerdos comerciales, el texto del acuerdo deja fuera otras políticas públicas que requieren protección en base al poder soberano de policía del Estado. $\mathrm{Tal}$ es el caso de otras medidas entorno a la protección del medio ambiente o la salud pública, como ser el control del consumo de bebidas

25 El borrador del Capítulo II - Inversión puede consultarse en: <http://trade.ec.europa.eu/ doclib/docs/2015/september/tradoc_153807.pdf> [consultado el 10.07.16].

$26 \mathrm{El}$ borrador el acuerdo puede consultarse en: <http://trade.ec.europa.eu/doclib/docs/2016/ february/tradoc_154329.pdf> [consultado 10.07.16].

27 Un estudio pormenorizado puede ser consultado en: SCHILL, Stephan. Reforming Investor-State Dispute Settlement (ISDS): Conceptual Framework and Options for the Way Forward. Documento de trabajo de The E15Initiative [en línea]. Ginebra: International Centre for Trade and Sustainable Development (ICTSD) y World Economic Forum, 2015 [consultado 29.03.2016]. Disponible en: <http://e15initiative.org/wpcontent/uploads/2015/07/ E15-Investment-Schill-FINAL.pdf> 
alcohólicas o el azúcar.

En suma, sería deseable que desde el Derecho de la integración también se traiga al tapete la discusión sobre la reforma del sistema de solución de controversias inversor-Estado, y de esta forma se den pasos firmes hacia un sistema regional y multilateral más justo para todos los Estados.

\section{REFERENCIAS BIBLIOGRÁFICAS}

ALVAREZ, José A. "Is the Trans-Pacific Partnership's investment chapter the new "gold standard"?" [en línea]. Working Paper IILJ 2016/3 MegaRed Series. Nueva York: New York University, School of Law, 2016 [consultado 01.07.16]. Disponible en: <https://wp.nyu. edu/megareg/wp-content/uploads/sites/3134/2016/03/Atvarez_IILJMegaReg_2016-3.pdf>

ARATO, Julián. "Corporations as Lawmakers". Harvard International Law Journal. 2015, vol 56, n² 2, p. 229-295.

BANIFATEMI, Yas. "The emerging jurisprudence on the Most-FavoredNation Treatment in investment arbitration". En: BJORKLUND, Andrea (ed.). Investment Treaty Law. Current Issues III - Remedies in International Investment Law: Emerging Jurisprudence of International Investment Law. Londres: BIICL, 2009, p. 241 - 273.

BAS VILIZZIO, Magdalena. "Arbitraje en el CIADI: algunas reflexiones en torno al caso Philip Morris contra Uruguay". En: TRAVIESO, Juan Antonio y CAMPI, Germán (ed.). Derecho Internacional Público. Su ingeniería y arquitectura en el siglo XXI. Buenos Aires: Editorial Albremática - elDial.com, 2016, p. 551-562.

BAS VILIZZIO, Magdalena. "Solución de controversias en los tratados bilaterales de inversión: mapa de situación en América del Sur" [consultado 01.07.16]. Revista de la Secretaría del Tribunal Permanente de Revisión. 2015, año 3, no 5, Marzo 2015, 233 - 253. Disponible en: $<$ http://www.revistastpr.com/index.php/rstpr/article/view/120/pdf>

BOLLYKY, THOMAS J. “TPP tobacco exception proves the new rule in trade. Council for Roreign Relations" [en línea]. Expert Brief, 4 de febrero de 2016. [consultado 09.07.16]. Disponible en: <http://www.cfr. org/trade/tpp-tobacco-exception-proves-new-rule-trade/p37509>

CIADI. Caso número ARB/10/7: Philip Morris Brand Sàrl, Philip Morris Products S.A. y Abal Hermanos S.A. contra Uruguay. Laudo del 8 de julio de 2016 [consultado 08.07.16]. Disponible en: <https://medios. presidencia.gub.uy/tav_portal/2016/noticias/NO_U130/Iaudo_spa1. pdf>

CORTE PERMANENTE DE ARBITRAJE. Caso 2012-2: Philip Morris Asia Limited contra la Mancomunidad de Australia. Laudo de jurisdicción y admisibilidad del 17 de diciembre de 2015. [consultado 16.05.16]. 
Disponible en: <https://pcacases.com/web/sendAttach/1711>

JOHNSON, Lise y SACHS, Lisa. "The TPP's investment chapter: entrenching, rather and reforming, a flawed system" [en línea]. CCSI Policy Paper. Columbia Center on Sustainable Investment [consultado 01.07.16]. Disponible en: <http://ccsi.columbia.edu/files/2015/11/TPPentrenching-flaws-21-Nov-FINAL.pdf>

MCHAFFIE, Kate. "New Zealand to implement plain packaging for tobacco" [en línea]. Lexology, 18 de febrero de 2016. [consultado 09.05.2016]. Disponible en: <http://www.lexology.com/library/detail. aspx?g=2f267230-efb7-4348-bb47-ee53c89bd601>

MICHELINI, Felipe. "The request for arbitration submitted by Philips Morris International vs Uruguay: a human rights perspective". Simposio Common Grounds New Zealand - América Latina - A legal Perspective (22 de octubre de 2015). Wellington: The Law School Victoria University, 2015.

ONU. Cuarto reporte del Experto Independiente sobre la promoción de un orden internacional democrático y equitativo. Documento A/70/285 [en línea]. Nueva York: ONU, 2015 [consultado 02.02.16]. Disponible en: <http://www.un.org/en/ga/search/view_doc.asp?symbo $\mathrm{l}=\mathrm{A} / 70 / 285 \& \mathrm{referer}=\mathrm{htt} \mathrm{p}: / / \mathrm{ww}$ w.ohchr.org/EN/Issues/IntOrder/Pages/ IEInternationalorderIndex.aspx\&Lang $=S>$

RANIERI, Agustina. "Deuda soberana: problemas y soluciones en la encrucijadă' [en línea]. Revista Pensar en Derecho. 2015, no 6, p. 245-304 [consultado 05.06.16]. Disponible en: <http://www.derecho. uba.ar/publicaciones/pensar-en-derecho/revistas/6/deuda-soberanaproblemas-y-soluciones-en-la-encrucijada.pdf $>$

SCHILL, Stephan. Reforming Investor-State Dispute Settlement (ISDS): Conceptual Framework and Options for the Way Forward. Documento de trabajo de The E15Initiative [en línea]. Ginebra: International Centre for Trade and Sustainable Development (ICTSD) y World Economic Forum, 2015 [consultado 29.03.2016]. Disponible en: <http:// e15initiative.org/wpcontent/uploads/2015/07/E15-Investment-SchillFINAL.pdf>

TAMBURINI, Francesco. "Historia y Destino de la Doctrina Calvo: ¿Actualidad u obsolescencia del pensamiento de Carlos Calvo?". Revista de estudios histórico-jurídicos. 2002, n XXIV, p. 81-101 [consultado 23.01.15]. Disponible en: <http://www.rehj.cl/index.php/rehj/article/ view/363/343>

TUNG, Ko-Yunng. "Investor-State dispute settlement under the TransPacific Partnership". The California International Law Journal. 205, vol 23, no 1, p. 19-25 [consultado 01.07.16]. Disponible en: <http://www. mofo.com/ /media/Files/Articles/2015/08/150800InvestorStateDisput eSettlement.pdf> 